\title{
MOBILE APPLICATIONS AS TOOL FOR EXPLOITING CULTURAL HERITAGE IN THE REGION OF TURIN AND MILAN
}

\author{
A. Rolando ${ }^{\text {a }}$, A. Scandiffio ${ }^{b}$ \\ a Dipartimento di Architettura e Studi Urbani, Politecnico di Milano, Milano, Italy - andrea.rolando@polimi.it \\ ${ }^{\text {b }}$ Scuola di Dottorato in Beni Culturali, Politecnico di Torino, Torino, Italy - alessandro.scandiffio@polito.it
}

KEY WORDS: Mobile application, GPS, Cultural heritage, QR code, Users interaction, Tracking technologies, GIS, Mapping.

\begin{abstract}
:
The current research aims at showing as applications working on personal mobile communication terminals such as smartphones, can be useful for exploration of places and, at the same time, as tools able to develop interaction between cultural heritage and users. In this sense, the use of smartphone applications can be combined with GIS in order to make a platform of knowledge useful to support research studies in the field of cultural heritage, with specific reference to accessibility issues and to the combined use of integrated technologies like GPS, QR code and GIS, with the final aim to find an useful methodology for collecting data by visitors and visualizing them through mapping techniques.

The research shows how the integration of different systems and technologies can be used as method for inquiring the interactions between users and cultural heritage in terms of accessibility to places. GPS devices can be used to record visitors movements (cultural routes) in terms of space and time; QR code can be used for users interaction with cultural heritage (tourists opinion, heritage ranking, facilities, accessibility); GIS software can be used for data management, analysis and mapping (tourist flows, more visited places). The focus of research is about a combination of information related to cultural routes with the information related to single cultural places. The focus of research is about a combination of information related to cultural routes with the information related to single cultural places. The current research shows the potential use of smartphone applications, as mobile device for collecting data, as means to record rides and more visited places by tourists. The research could be divided into three steps; the first one concerns with GPS that can be used to record routes; the second one deals with interaction between tourists and cultural heritage through a system based on QR code; the third one is about GIS, used as tool for management, analysis and visualization of data flows. In the current research, the field of investigation refers both to the territorial scale of Turin - Milan axis, and to the local scale of small cities localized in the territories in between. The research has been applied to Turin - Milan infrastructural axis, with the aim to represent the relationships that can be established between mobility infrastructure and cultural heritage. Such relationships should be intended in terms of accessibility from mobility infrastructure (motorway exit, service areas, railways stations) to cultural heritage localized in the surrounding landscape. The richness of cultural heritage and landscape along the chosen infrastructural bundle represent a great opportunity for territorial development in terms of attractiveness, both for local inhabitants and for tourists. Nowadays, the use of tracking technologies can be applied to investigate tourist flows, behaviors of local inhabitants in the historic city centre, number of visitors in the city and so on. In this sense it is possible to apply these technologies, which are particularly relevant in urban studies, extending them to the territorial scale of the Turin - Milan region.

The large amount of available geo-referenced data can be used in different ways and it is very potential for different kind of analysis: it is possible to show tourist flows in the territory, receive information about more visited places, obtain interaction from users and cultural heritage in terms of visitors opinion about the places, give information to tourists about cultural places, monitor the accessibility to the places, understand the use of means of transport and keep under control the impacts of tourism (social, cultural, environmental) on territory. Applications based on smartphones can be considered a powerful device for visitors but also for institutions that are involved in tourism and cultural heritage management. In fact, the use of mobile applications it can produce a real time data exchange between geographical position of users and system that receives data.

The research shows the opportunity to use GPS and QR code, integrated in a single smartphone application, with GIS software. In the current step of the research only the interaction with GPS and GIS has been tested on the case study of Turin - Milan. Further development of research could be realized to test the real integration of QR code with other systems, developing an application that supports QR code and GPS, and installing a barcode or other interactive devices such as NFC (Near Field Communication) on each relevant cultural places. It is evident that different actors, such as tourists, cultural heritage institutions and employers, have to be involved in this process, in order to have a deeper understanding of the problem, also integrating bottom up and top down contributions.
\end{abstract}




\section{INTRODUCTION}

\subsection{General framework}

The current research deals with smartphone applications based on mobile phones as new tools for recording information produced by people movements in the territory. The connection between GPS based on mobile phone and smartphone applications represents a powerful tool in terms of data collection, that could be used to make new territorial analysis in the field of cultural heritage and landscape issues, as a new layer of knowledge that could be added to the traditional ones. The great amount of georeferenced data that comes from GPS based on smartphone applications could be used in the field of heritage exploitation research in order to understand visitors movements and more visited places.

This research has to be framed within general territorial studies that have been carried on Turin - Milan infrastructural axis in the last few years (Rolando, 2006), with aim to develop new strategies to increase the connectivity and accessibility of cultural heritage in relation to the mobility infrastructures. In that sense, the research has been applied to the infrastructural axis between Turin and Milan with aim to enhance the territorial role of the territories in between the two main cities, characterized by a very complex landscape in terms of stratification of different layers and human actions. In this perspective the territories in between Turin and Milan are already relevant in terms of integration between cultural heritage and agricultural landscape, but need, at the same time, to be better connected to the main infrastructure, that cross this territory, in order to improve the accessibility to and from places, that physically are already existing, and need to be enhanced. The main goal is to design of a slow network of walking/cycling paths that could be useful to increase the level of accessibility of these places, giving new values to the exiting cultural heritage. Nowadays it could be necessary an improvement of existing connections starting from the main infrastructure, encouraging the accessibility from the main gates located along the route (motorway exits, service area, railways stations) to cultural places, in order to develop a system of slow network that connect different places and support the development of new local tourism in this region. The current research focuses the attention on the main infrastructural nodes, that could be rethought, in a more effective way, in order to connect places localized in the surrounding landscape of infrastructure through a system of walking/cycling paths (Rolando et al., 2013). According to this approach, it could be useful investigate new methods and techniques that can support decision makers to define which places and routes are more relevant than others, in terms of tourist attractiveness and physical accessibility. The combined use of GPS tracking, based on smartphone applications, and GIS could represent an interesting platform of knowledge, in terms of new tools and techniques for territorial analysis, to reach these goals. In the last decades many research activities have been developed about the application of tracking technologies to urban environment and urban cityscape, with the aim to use GPS data, as new layer of knowledge for urban studies and urban analysis (Van Der Spek et al., 2009). Most of them are, particularly, related to analyze pedestrian movements in the city and their relationships with urban structure. However, specific research studies and urban simulations have been developed at urban scale (Van Der Hoeven et al., 2008). Other research studies analyze spatial tourist behaviours in the historic city centre, with the aim to identify the most attractive areas in the cities, in a touristic perspective, but also investigate which kind of relationships could be established between touristic movements and urban pattern, in relation to availability of touristic services and urban infrastructure. In this case, the research developed a methodology to track movements in historic city centres, with the aim to investigate touristic potentiality of the site, in relation to visitors needs (Shoval et al., 2008). Other research activities are related to the use of cellular phone data and their mobile phone positioning, used to estimate people movements in the cities in a dynamic way, giving new visions and images of cityscape (Ratti C. et al., 2008). The large amount of data could be useful to analyze tourism flows and to define new territorial marketing strategies, but also to manage the impacts of tourism at spatial level, including social and environmental issues (Shoval et al., 2010).

\subsection{Challenge}

The increasing use of GPS tracking based on smartphone applications could be applied to investigate the level of cultural heritage accessibility at territorial scale. The main challenge of this research is to combine the use of GPS with GIS, showing the potential interoperability between them and their real application to heritage accessibility studies. The current research has been not applied to urban environment, but to the territories in between the main cities and particularly to extra urban space. Therefore, the use of GPS is a powerful tool to support territorial investigation and to analyze the problem of accessibility at territorial scale. This issue is strongly related to the specific scale of the territories analyzed, this represent a crucial point, in the sense that contents are very different from urban scale. The main goal is to define a method that could be useful to establish which places and routes are more relevant than others, evaluating the amount of tracks that users record with their own device. GPS based applications seem to represent the best tool to collect territorial information produced by users. Instead, GIS is the platform that allow to make analysis on .gpx files in terms of visualization and mapping of this data. The expected results should reveal a selection of routes and places that could represent the more tracked paths and visited sites. The so popular use of GPS on smartphone application represent a very potential tool in a quantitative (big amount of data) and qualitative (georeferenced data) way, that can be easily used by users easily. Georeferenced data, related to bicycle or pedestrian rides, can be recorded by smartphone. In that sense, more attractive places and more frequented routes could be established, evaluating the overlapping of different tracks and their intersection. The second step of the work concerns with matching the tracks layer and the physical places layer. A further research development is aiming at evaluating the correspondence between the routes that are more intensively tracked and the quality of the physical landscape. The aim of this work is to use the personal experience of users, as a local trip in the landscape, to establish a ranking of places and routes that could be redeveloped according to the accessibility from the main infrastructural gates. This challenge could represent a strategy to give again new values to the places and to the routes that cross the landscape, as new direct experience of knowledge.

\section{METHODS AND TECNIQUES}

\subsection{GPS and GIS as platform of knowledge}

The common use of GPS on smartphone applications could increase the availability of georeferenced data, coming from bike or walking rides. GPS is already available on most 
personal mobile smartphone, fact that makes possible to record a large amount of georeferenced data from different kind of users. The use of GPS could be related to measure the spatial movement of users, but also to calculate the time spent in the different locations in relation to the density of points per second (Shoval et al., 2007) (Van Der Spek et al., 2009). In the current research GPS has been applied to describe spatial movements, and mainly to analyze which physical places are frequented by visitors during a local trip. Different kind of tracks, starting from the main gates along motorways (motorway exits and service area) and railways (railways station) have been recorded by GPS. Various cycling tracks have been recorded by users in the surroundings of motorway A4 between Turin and Milan through GPS based on mobile phone. Therefore, GIS represent the best platform to visualize .gpx files with different colours, in order to make evident the differentiation of the tracks (Figure 1) (Nijhuis, 2008). The analysis could be conducted in relation to the routes and to the places, as two different topological entities. More frequented routes can be represented by tracks overlapping with a red colour gradient that shows the different level of tracked routes. The use of gradient, in terms of visual representation, shows clearly which are the most frequented routes and which is the favourite route by users. In that sense, it could be interesting to investigate why a route is more significant than others in relation to landscape structure (Figure 2). The most relevant places can be established by intersection of different tracks. A selection of more frequented routes and more relevant places has been shown in Figure 3. The intersection points, between two o more routes, can represent a significant point, that could be interpreted as an attractive place, in terms of recognized point of interest, or as new place of interest frequented by a lot of users.

It is possible to investigate the correlation between more tracked routes and physical characteristics of the routes, in order to understand the different factors that could influence the choice of route and users' behaviours. Of course, the interpretation of users' choice could be in terms of space, in the sense that users prefer, maybe, the shortest route to go from A to B. The second factor could be represented by time spent to reach the final destination. Another factor could be represented by the level of comfort that could be related to other parameters, such as the steepness of route. The presence of heritage hotspots, along the routes could represent another objective parameter, in terms of touristic attractiveness. Time, space, comfort and heritage sites can be measured by four objectives parameters, that, respectively, correspond to minutes, kilometres, average steepness of routes and number of heritage sites that routes intercept. This kind of analysis combine objective parameter with others subjective factors that could influence the choice of a particular path than others. It possible to identify the level of pleasantness in the trip that could be related to spatial quality of landscape crossed by routes. The level of safety could be considered as subjective parameter, in relation to lighting of route or the presence of public services that offer assistance to users. Spatial quality of landscape and the level of safety could be measured by a range of values that correspond to different level of that parameters. This kind of investigation could be add to the current research, that has been based on the use of tracks as tool to realize new analysis in the field of cultural heritage and landscape at territorial scale. It could be really interesting to add a parametric analysis to the current one, analyzing the problem of cultural heritage accessibility in a broader way.

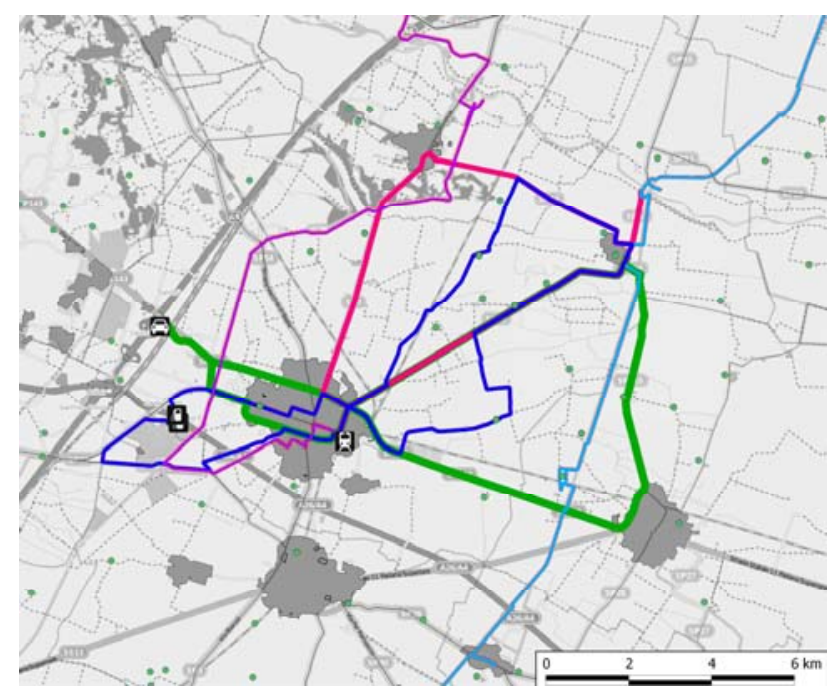

Figure 1. Map of different cycling tracks recorded by GPS

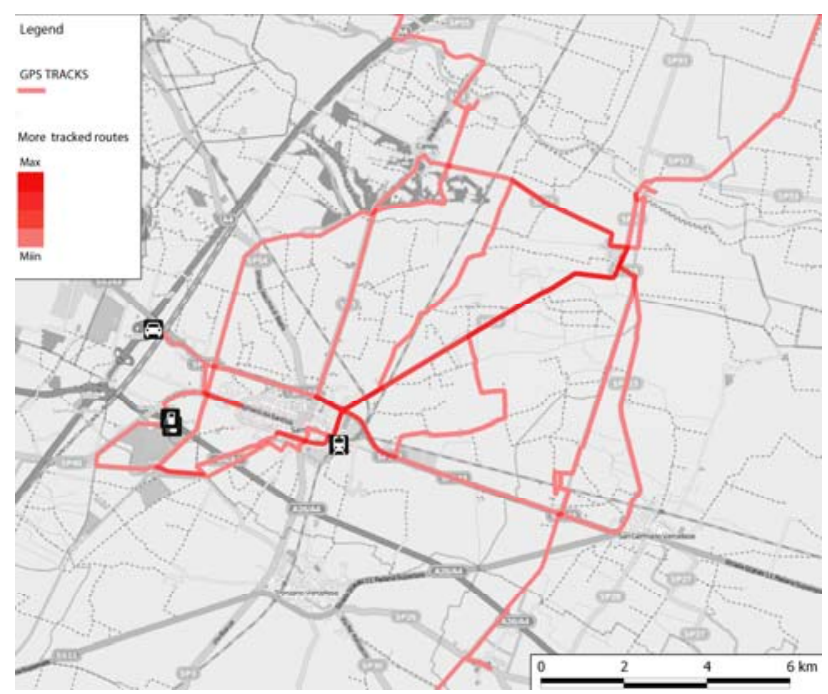

Figure 2. Map of gradient about more frequented routes

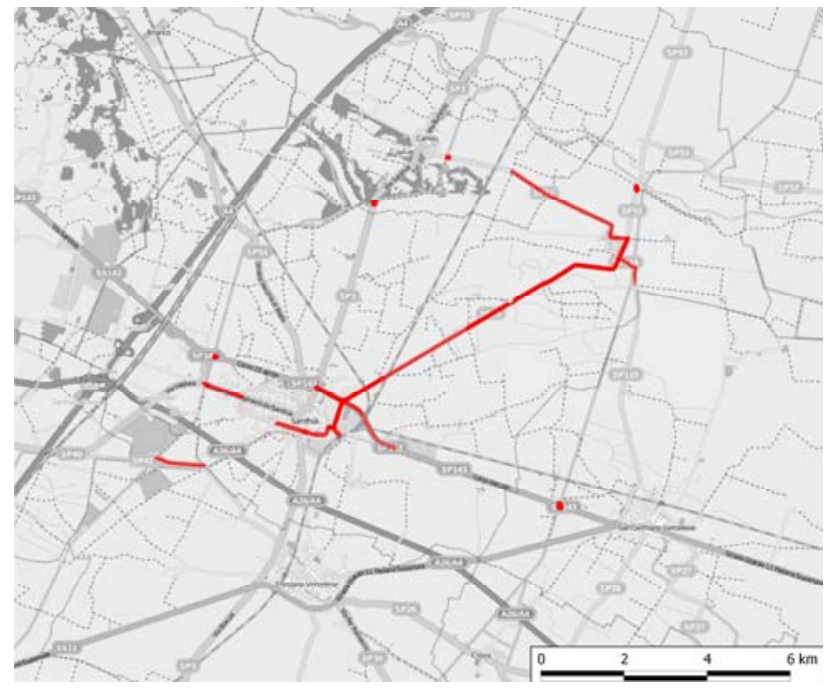

Figure 3. Map of more frequented routes and tracked places 


\subsection{GPS tracks as tool to establish "new places"}

The most relevant cultural places can be established by evaluating the correspondence between GPS tracks and physical places. In terms of methodology, tracks layer could represent a new layer of knowledge that can contribute to the investigation of cultural heritage accessibility. It is interesting, from methodological point of view, matching heritage sites layer with tracked routes layer; result layer represent the combination between more tracked routes and physical positions of heritage sites (Figure 4).

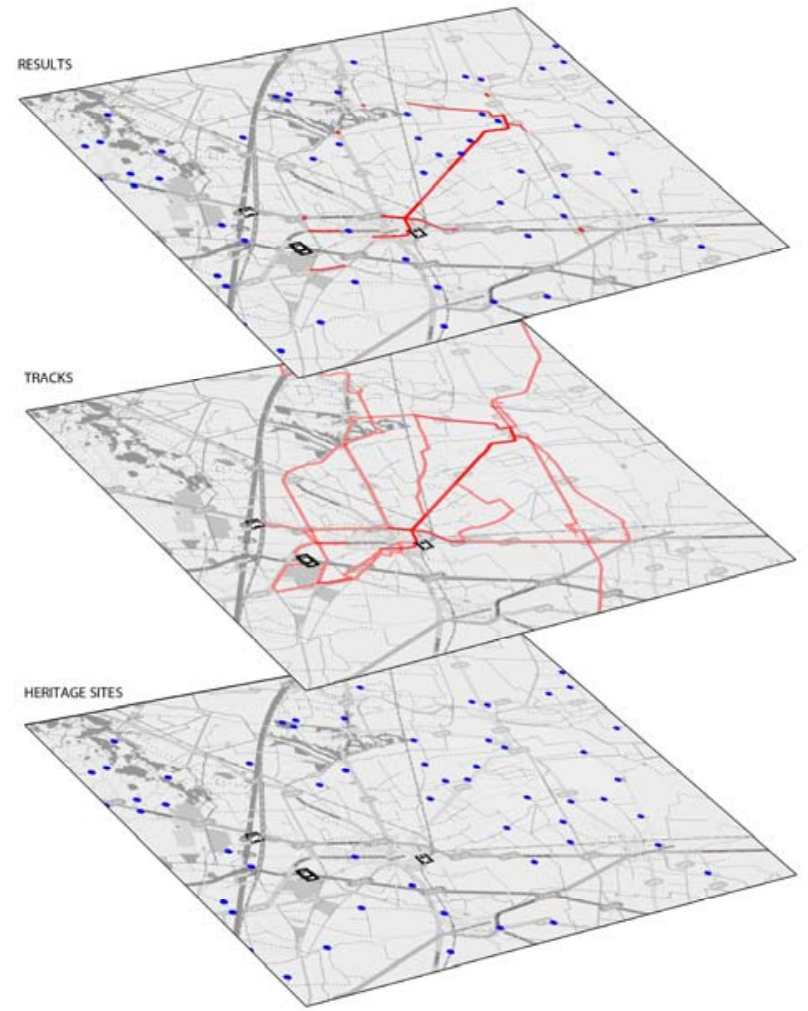

Figure 4. Different layers of knowledge: heritage sites, tracked routes and

In that sense, tracking technologies can show new types of relationships between physical places and routes. It is possible to investigate two kind of results. In this sense, it is interesting to understand the role of tracked routes that show the physical connection between city centre and external cultural heritage. It is possible underline that the most tracked route is also related to the presence of a certain number of heritage sites, that are distributed in the surrounding landscape. A density map of heritage sites shows a sort of densification of heritage sites, distributed in the surroundings of more tracked routes (Figure 5). So the main question is about the possibility to establish a direct relationship between physical position of heritage sites and more tracked routes. It can be assumed that the physical presence of the cultural heritage is not enough to suppose that a direct correlation exists between heritage sites and tracked routes. A deeper analysis could inquiry the importance of different heritage places that probably according to the different relevance attributed by visitors (bottom up users' contribution). The second outcome deals with "new places" that could be established by the intersection of different tracks (Figure 6). The current research tries to answer to the following questions: which is the role of these new points/places in relation to the tracks? Is it possible to consider them as meaningful places? Is it possible to establish a correspondence between them and physical places? Certainly, the intersection between different tracks could represent a significant point, in the sense that they have more value than simple crossing points. Therefore, it could be established a hierarchy of points: heritage hotspots represent a cluster of points that are usually recognized by people and clearly represent a category of attractive points. New points, obtained by tracks intersection, could assume a new role in territorial dynamics. When these points correspond to high spatial quality places, they can assume more relevance in terms of attractiveness for visitors. In the current research, new places correspond to the intersection between two important canals for irrigation (Cavour canal and Naviglio di Ivrea), between a river and a canal (Elvo river and Cavour canal), or between an historic route and a canal (via Francigena and Cavour canal) that are important elements in the landscape, and could be added to the layer of points of interest for visitors. In the case that intersection points have not a physical correspondence with quality of places, they can be used in other way, such as info point, services, facilities etc. In this case intersection points could assume a new relevance in terms of amount of people that cross those places.

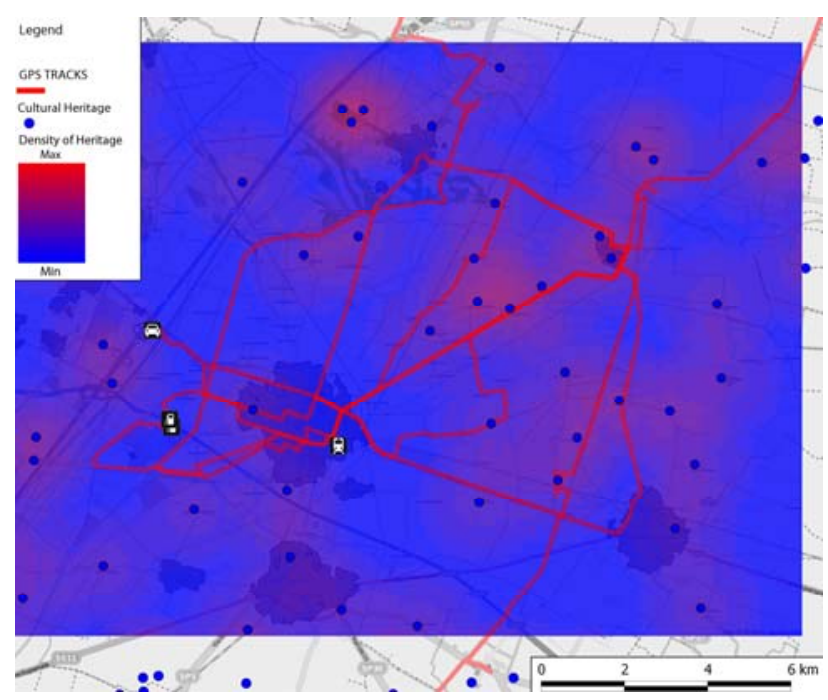

Figure 5. Density map of heritage sites and more tracked routes

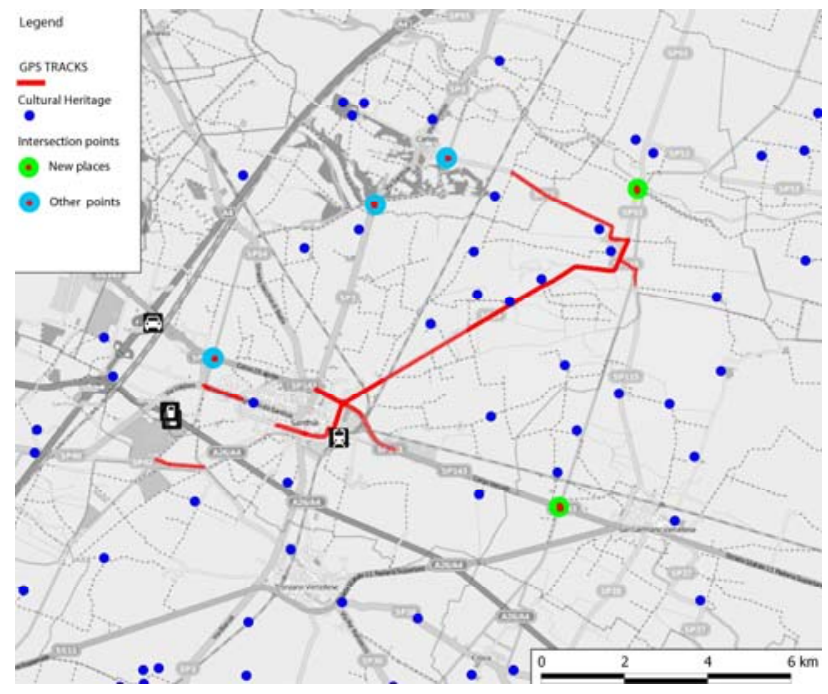

Figure 6. Map of new places 


\subsection{QR Code as future development for research implementation}

The combined use of QR code and GPS in a smartphone application could increase the potentiality of these tools for spatial analysis, in relation to the different type of data that could be collected. GPS and QR code could improve the interaction between users and cultural place developing a sort of information exchange between them. In this case, information flow would be not only in terms of passive exchange of information between users and smartphones, but also as active communication channel between users and physical cultural places. Therefore, GPS tracking represent a passive method for collect information by people movements; QR code is, instead, a different technology, that is able to read 2d-barcode or matrix barcode, that are, physically, installed on the physical objects. In the current research barcode could be installed on the existing cultural heritage, in order to obtain quantitative and qualitative information from users. More heritage information could be easily given to users on the physical place. Users could express their opinion on the pleasantness of the places and contribute to the improvement of system. On the other side, it could be possible monitoring the number of visitors and the accessibility to the places. Therefore, the combined use of GPS and QR code based on smartphone application can increase the amount and quality of data available for research studies.

\section{CONCLUSIONS}

The current research tried to investigate how new devices and technologies, related to widely diffused tools for geo positioning and route tracking, could be applied in spatial analysis with specific focus on cultural heritage and landscape issues. In the field of cultural heritage, new layers of knowledge can be added to the traditional ones, giving new points of view to this subject.

The first point deals with the scale of investigation, in relation to cultural heritage issue, that correspond to territorial one. In this sense, the research carried out a vision of cultural heritage as complex system that can be analyzed a territorial scale using new tools and technologies.

The second point is about the contribution of these technologies to cultural heritage studies. It could be relevant considering the use of smartphones as an interface between users and cultural heritage, but also as mobile tool for exploring landscape. In this sense, the research tried to analyze the relationships between visitors movements and fixed places in the landscape. New topological entities can be discovered considering users movements: points/places and tracks/routes.

Finally, the combination of different technologies, such as GPS and GIS, can contribute to make available a new platform of knowledge to investigate spatial configuration of cultural heritage in relation to their accessibility at territorial level.

\section{REFERENCE}

Nijhuis S. 2008. Application of GPS data in geographic information system. In: VAN SCHAICK J. et al. Urbanism on track. IOS Press. Amsterdam.

Palma R., Occelli C., Sansone M. 2012. La ciclostrada del Canale Cavour. Torino. Regione Piemonte Editore.

Ratti C., Sevtsuk A. 2008. Mobile surveys. In: VAN SCHAICK J. et al. Urbanism on track. IOS Press. Amsterdam.

Rolando A. 2006. La configurazione di un nuovo paesaggio lungo la linea ad alta capacità tra Torino e Milano. In: PRATELLI, Alberto. Sui codici del disegno di progetto. Udine: Editrice Universitaria Udinese, 2006, pp. 119-136.

Rolando A., Scandiffio A. 2013. Milan-Turin: a bundle of infrastructures to access a network of places, between cultural heritage and landscape. In AA.VV. Proceedings of S.A.V.E. Heritage, XI International Forum le vie dei Mercanti. CapriNapoli. La Scuola di Pitagora.

Saeed G., Brown A., Knight M., Winchester M. 2010. Delivery of pedestrian real-time location and routing information to mobile architectural guide. Elsevier. Automation in Construction n. 19 pp. 502-517.

Shoval N., Isaacson M. 2007. Tracking tourists in the digital age. Elsevier. Annals of Tourism Research, Vol. 34, n. 1, pp. 141-159.

Shoval N. 2008. Tracking technologies and urban analysis. Elsevier. Cities n.25, pp.21-28

Shoval N., Isaacson M. 2010. Tourist mobility and advanced tracking technologies. New York: Routledge.

Van Der Hoeven F. D., Van Schaick J., Van Der Spek S.C., Smit M.G.J. 2008. Urbanism on track: Application of tracking technologies in urbanism. IOS Press. Amsterdam.

Van Der Spek S., Van Schaick J., De Bois P., De Haan R. 2009. Sensing Human Activity: GPS Tracking. Sensors. pp.30333055. 